\title{
REVISIONES
}

\section{Bases conceptuales del enfoque histórico-cultural para la comprensión del lenguaje}

\author{
Conceptual bases of historical-cultural approach to the understanding of language \\ Bases conceituais do enfoque histórico-cultural para a comprenção da linguagem
}

\section{Sílvia Ester Orrú}

Faculdade de Educação - Universidade de Brasília - Brasil. Correo electrónico: seorru@unb.br

\begin{abstract}
RESUMEN
El propósito de este artículo es presentar los fundamentos conceptuales del enfoque histórico-cultural de Vigotsky para la comprensión del lenguaje, a saber, referidos a la propia especie humana, y comprender al individuo como un desarrollo social y cultural en una historia, que parte de lo interpersonal a lo intrapersonal y que tiene al lenguaje como mediador de todas sus relaciones. También destacan las contribuciones de este enfoque a la educación de las personas con necesidades educativas especiales, concentrándonos en la persona como sujeto social que se construye en las relaciones sociales, culturales e históricas, a través de la mediación de otro sujeto y de las señales que se producen en esta mediación, el lenguaje, principalmente. Se concluye que, en vista del enfoque históricocultural, una propuesta para la persona con necesidades especiales se debe basar en el contexto de las relaciones personales, las actitudes y sus alrededores, habilitando al educador a través de la acción mediadora de los signos.

Palabras clave: enfoque histórico-cultural, lenguaje, personas con necesidades especiales.
\end{abstract}

\begin{abstract}
The following article, as a result of our PhD thesis, intends to present the conceptual basis of Vigotsky's culturalhistorical approach for the comprehension of language, proper to the human species. To understand the individual as a social and cultural being in a developmental history, that goes from interpersonal to intrapersonal, and language being the mediator of all his/her relations. Likewise, to highlight the contributions of this approach for the education of people with special needs. To stress that this person is a social individual that develops through social, cultural and historical relations by the mediation of another individual and the existing signs in this mediation, the language being the main sign. We can conclude that, from the cultural-historical approach, a leap is expected in the development of the individual with special needs in the personal relations context, in the teacher's enabling and engaging attitudes and the mediating action of signs.
\end{abstract}

Key words: cultural-historical approach; language; people with special needs.

\section{RESUMO}

Apresentam-se os fundamentos conceituais do enfoque histórico-cultural de Vigotsky para a compreensão da linguagem, própria do ser humano. Compreender o sujeito como um desenvolvimento sociocultural que parte do interpessoal para o intrapessoal, tendo a linguagem como mediadora de todas as suas relações. Também se destacam as contribuições de este enfoque para a educação de pessoas com necessidades educacionais especiais. Enfatiza-se que esta pessoa é um sujeito social que se constrói nas relações sociais, culturais e históricas por meio da mediação de outro sujeito e que a linguagem é o principal sinal em esta mediação. Conclui-se que, em razão do enfoque histórico-cultural, é esperado um salto a ser dado pelo sujeito com necessidades especiais, a partir do contexto de relações pessoais, das imediações possibilitadas pelo educador e pela ação mediadora dos signos.

Palavras chave: abordagem histórico-cultural, linguagem, pessoas com necessidades especiais. 


\section{INTRODUCCIÓN}

Después de años de estudios sobre el comportamiento, la personalidad y la constitución subjetiva de la persona, los estudios de la psicología, a partir de una perspectiva histórico-dialéctica del marxismo, han tratado de demostrar que la génesis de las funciones psicológicas se encuentra en la interacción socio-cultural y no en una jurisdicción endógena. Vigotsky (1994), hace más de 50 años, declaró que el desarrollo cultural del niño se da primeramente en el plano social y después a nivel individual, en el interior del propio niño. Explica así esta génesis:

Primero el individuo realiza las acciones exteriores, que serán interpretados por los que le rodean, de acuerdo con los significados culturalmente establecidos. A partir de esta interpretación es que le será posible al individuo atribuir significado a sus acciones individuales $y$ desarrollar sus propios procesos internos psicológicos, que pueden ser interpretados por el mismo partiendo de los mecanismos establecidos por el grupo y comprendido a través de los códigos culturales compartidos por los miembros de este grupo (Vigotsky, 1994: 15).

En este sentido:

Todas las funciones en el desarrollo del niño aparecen en dos momentos: primero a nivel social, y después a nivel individual; en primer lugar entre las personas (inter-psicológicamente), y después en el interior del propio niño (intrapsicológicamente) [...]. Todas las funciones superiores se originan a partir de las relaciones reales entre los individuos humanos (Vygotsky, 1994: 75).

Para Vigotsky, las funciones psicológicas superiores deberían ser comprendidas en las relaciones sociales presentes en la vida del individuo, siendo el hombre un participante activo en el proceso de creación de su medio ambiente, y no determinado por éste. El hombre es un ser social y cultural en una historia que se desarrolla, que parte de lo interpersonal a lo intrapersonal, teniendo al lenguaje como mediador de todas sus relaciones.

\section{LA ACTIVIDAD CONSCIENTE DEL HOMBRE SEGÚN EL MARXISMO}

Los fundamentos respecto al origen de la actividad consciente del sujeto, deben ser buscados en las condiciones sociales de vida históricamente producidas que posibilitan cambios estructurales en el comportamiento. Por lo tanto, los motivos biológicos de la conducta, los motivos superiores y las necesidades aparentes acompañan el comportamiento que se subordina a la percepción inmediata. Las formas superiores de comportamiento como resultado de la abstracción de las influencias inmediatas del entorno, en conjunto con "los programas de comportamiento consolidados por vía hereditaria, y la influencia de la experiencia pasada del propio individuo", proporcionan el nacimiento de la transmisión y asimilación de la experiencia de toda la humanidad como una tercera fuente de la formación activa (Lúria, 1979: 75).

Encontramos, en la ciencia histórica, el trabajo social, la utilización de los instrumentos de trabajo y también en el surgimiento del lenguaje a los factores fundamentales, que nos hablan acerca del pasaje de la historia natural animal para la historia social del hombre, contribuyendo con el emerger de la conciencia. Durante su historia, el ser humano tanto se valió de los instrumentos de trabajo, como también los perfeccionó conforme sus 
necesidades de supervivencia, distinguiendo de esta manera su actividad de la conducta animal, ya que para ésta, era necesario valerse de un elaborado y complejo proceso de sentidos y significados, lo que requiere de una actividad consciente.

Percibir la necesidad de elaborar sus instrumentos de trabajo interfiere completamente en la estructura de comportamiento del ser humano, pues esto es una actividad compleja en favor de una necesidad que precisaba ser apurada y que, en el caso del individuo primitivo, era su alimentación. En el pasaje de la historia natural del animal a la historia social del hombre, hay un momento en que el hombre ya no actúa más impulsado por motivos biológicos, sino por acciones propias y exclusivas, regidas por los objetivos alcanzados y la consciencia adquirida, conforme la obtención de resultados concretos.

En la medida en que el hombre lleva a cabo varias acciones para alcanzar una objetivo determinado, su comportamiento se transforma, apareciendo así una actividad propia y consciente, y que por lo tanto viene a influenciar sobre nuevas formas de comportamiento, que no se conducen más por motivos biológicos, sino por una actividad consciente, producto de las nuevas formas histórico-sociales.

Como segunda condición para la formación de la actividad consciente del ser humano está la formación del lenguaje. Por un tiempo, la psicología no dio la merecida atención al lenguaje como un factor fundamental en la formación de los procesos mentales del niño. Las actitudes se orientaban por el pensamiento bejeveurista de Thorndike, Watson y Guthrie, en el que se describen las formas complejas de la actividad del niño como originarias de un condicionamiento de hábitos en el que se determinaba al lenguaje como un aspecto consecuente del desarrollo de los hábitos motores, sin hacer ningún tipo de relación específica de éste con la conducta y la formación intelectual del individuo.

Juntamente con esta concepción mecanicista, surgió el pensamiento idealista, que comprendía al desarrollo de los procesos psíquicos como originarios de una fuente o actividad interna en el que el lenguaje estaba lejos de ser contemplado como una parte del complejo de actividad y desarrollo de las capacidades intelectuales del niño. Sin embargo, ya en el año de 1934, Vigotsky presentó el lenguaje como decisivo en la formación de los procesos psíquicos, ejecutando varios experimentos referentes a la formación de la atención activa, donde observó que, en los procesos de desarrollo de la memoria, que acontecían a través de la adquisición del lenguaje, la memorización se manifestaba de manera activa y voluntaria.

Como ya fue expuesto, de acuerdo con Lúria (1979: 78) "era común entender al lenguaje como un sistema de códigos mediante los cuales se designaban los objetos del mundo exterior, sea por sus acciones, cualidades, o las relaciones entre ellos, etc". En el lenguaje, las palabras califican y caracterizan tanto las acciones como a los objetos. Este lenguaje, que consiste en palabras que se unen en frases, constituye el principal medio de comunicación del hombre, utilizado para que las informaciones y experiencias sean aprendidas, conservadas y transmitidas de generación en generación.

\section{LAS CONTRIBUCIONES DE LÚRIA PARA EL LENGUAJE}

Con respecto al lenguaje, éste es comprendido por Lúria (1987) como un complejo sistema de señales convencionales que representan objetos, acciones, características o relaciones y posibilitan la transmisión de los conocimientos constituidos en el proceso 
histórico-social y de importancia fundamental en lo que se refiere al desarrollo de los procesos cognitivos y de la conciencia del ser humano, actuando, inclusive, como un puente entre conocimiento sensorial y el racional, como un proceso de continua concientización, constituido por medio de las formas sociales de la experiencia histórica humana.

La palabra realiza para el hombre, el grande trabajo del análisis y clasificación de los objetos que fue formado a través del largo proceso de la historia social. Esto dio al lenguaje la posibilidad de ser no sólo un medio de comunicación, sino también el vehículo más importante del pensamiento, que asegura la transmisión de lo sensorial para lo racional en la representación del mundo (Lúria, 1987: 81).

Es aceptada, comúnmente, la afirmación de que el ser humano es un ser consciente de sus acciones, no obstante, por más que se comprenda que es el único inmerso en un contexto histórico, social y cultural, en donde surgen sentidos y significados, existen corrientes de pensamiento que no pocas veces entran en conflicto con esta opinión, alegando que los motivos biológicos son la mejor explicación para una comprensión científica del comportamiento humano.

El hombre es el único ser capaz de asimilar la experiencia que no es propiamente suya y de transmitirla a otros individuos de generación en generación. La imitación es, por tanto, limitada en la formación del comportamiento animal y se transmite de una manera práctica y directa, derivada de la propia experiencia. En los seres humanos, sin embargo, la transferencia de la experiencia se produce de manera articulada por las informaciones acumuladas a través de la historia de las generaciones pasadas.

De esa asimilación de experiencias materiales o intelectuales, es que se caracteriza la historia social del hombre, de ahí emana su actividad consciente, la que, según Lúria (1979), puede explicarse a través de tres características fundamentales.

En primer lugar, la actividad consciente del sujeto no está necesariamente ligada a motivos biológicos, ya que la mayoría de nuestras acciones no depende de las necesidades biológicas. Por el contrario, la actividad humana está regulada por necesidades más complejas que habitualmente son conocidas como intelectuales o superiores. Entre ellas, se encuentran "las necesidades cognitivas, "instando al hombre a adquirir nuevos conocimientos, la necesidad de comunicarse, la necesidad de ser útil a la sociedad y de ocupar en esta una posición determinada, etc" (Luria, 1979: 71-72). La actividad consciente del hombre va más allá de las cuestiones biológicas, pues muchas veces se encuentra en confrontación directa con ellas, llegando inclusive a ignorarlas o, contrariándolas, colocarse en situaciones de riesgo por causa de la autonomía de su comportamiento en relación a los motivos biológicos.

En segundo lugar, la actividad consciente del hombre no se encuentra delimitada por los efectos causados por estímulos externos sobre la mente o los sentidos, como resultantes de experiencias individuales súbitas. El hombre tiene la capacidad de pensar y reflexionar sobre las condiciones del medio circundante, de manera mucho más profunda e intensa que los animales. Por esta razón le es posible separar conscientemente sus pensamientos y sentimientos por algún objeto o acontecimiento y también superar las relaciones de dependencia y de vínculo con las cosas o experiencias vividas eventualmente. A partir de esto, es capaz de interpretar y de tomar tales fatos como referencias legítimas y racionales acerca de las impresiones exteriores. Por lo tanto, se puede entender el claro fundamento que afirma, que el comportamiento humano es libre, cuando está fundamentado en el reconocimiento de la necesidad. A este respecto, Lúria comenta: 
Al salir de paseo en un día claro de otoño, el hombre puede llevar un paraguas, pues sabe que el tiempo es inestable en otoño. Aquí sigue un conocimiento profundo de las leyes de la naturaleza y no la impresión inmediata de un clima soleado y cielo despejado. Sabiendo que el agua del pozo está envenenada, el hombre nunca irá a beberla aunque tenga mucha sed; en este caso su conducta no se guía por la impresión inmediata de la atracción por la agua, mas por el conocimiento consciente de la situación (1979: 73).

El tercer aspecto mencionado por Lúria (1979: 73), como trazo característico que distingue la actividad consciente del hombre del comportamiento animal, es la asimilación de la experiencia humana que está basada en el proceso de aprendizaje de su historia social, la acumulación de conocimientos y de habilidades adquiridas que se transmiten de generación en generación; y esto difiere claramente del comportamiento animal, que se yergue sobre dos principios: 1) Un programa de comportamiento hereditario, intrínseco en su genotipo; 2) los resultados de la experiencia individual directa.

Desde muy temprano, el niño construye su comportamiento a partir de la influencia de lo que sucede a su alrededor, y va asimilando las diversas habilidades que fueron construidas en el proceso de la historia social. A través del habla, las otras personas le repasan los conocimientos primarios y un tiempo después, por medio del lenguaje, será capaz de asimilar las más importantes adquisiciones de la humanidad en el espacio escolar. Gran parte de los conocimientos, habilidades y formas de proceder son el resultado de las adquisiciones asimiladas de la experiencia histórica y social de generaciones anteriores. Éste es el componente principal que distingue la actividad consciente del hombre del comportamiento animal.

La psicología que se fundamenta en el marxismo sostiene que las características peculiares del hombre se pueden encontrar en una perspectiva histórico-social de la actividad, relacionada al trabajo social, al desarrollo de los instrumentos de trabajo y al emerger del lenguaje. Con los animales no ocurre de la misma manera, pues toda la actividad psíquica parte de las condiciones de supervivencia de las formas de vida para su orientación en el medio ambiente.

\section{LA ACTIVIDAD CONSCIENTE DEL SUJETO Y EL LENGUAJE}

De acuerdo con la teoría marxista, el origen del lenguaje es posible gracias a las condiciones sociales de trabajo, cuyo inicio se refiere al período de transición de la historia natural para la historia humana, enfocando en la necesidad de compartir y transmitir a las generaciones, las informaciones y experiencias previamente asimiladas.

En cuanto la lengua se construyó como un sistema de códigos independientes que nombraba objetos, acciones y características, sirviendo como medio de transmisión de informaciones, el lenguaje, por su parte, fue clave para que más tarde el hombre organizase su actividad consciente, dando lugar a la formación de la conciencia. Así, a lo largo de la historia, la actividad consciente del hombre pasó por transformaciones, de las cuales Lúria (1979: 80-81) destaca tres:

1) Al nombrar los objetos y eventos del mundo exterior con palabras aisladas o combinadas, el lenguaje proporciona la discriminación de estos objetos, posibilita que dirijamos la atención hacia ellos y que los conservemos en la memoria, haciendo posible que trabajemos con sus imágenes, mismo que no estén de forma presente. El lenguaje 
“duplica el mundo perceptible" pues preserva la información que llega del mundo exterior y permite la creación de un mundo de imágenes interiores.

2) Las palabras de una lengua denominan cosas como también abstraen sus peculiaridades. Relacionan lo que es perceptible a determinadas categorías y garantizan el proceso de abstracción y de generalización como factor contribuyente para la formación de la conciencia. Por ejemplo, "la palabra reloj indica que este objeto sirve para marcar las horas, la palabra mesa indica que este objeto sirve para ser cubierto". Ambas palabras indican también todas las propiedades de estos objetos, sin importar su apariencia o su tamaño. La palabra, por lo tanto, abstrae los indicios del objeto y generaliza los diferentes objetos según sus características exteriores, agrupándolos por categoría, transmitiendo al hombre de esta manera la experiencia de las generaciones anteriores. La palabra opera, mediante el hombre, la acción de analizar y clasificar los objetos que fueron siendo construidos con el recorrer de la historia social, haciendo posible que el lenguaje, además de ser una forma de comunicación, también se transformase en el instrumento más importante del pensamiento en la transición de la percepción sensorial para la representación racional del mundo.

3) Como instrumento fundamental para la transmisión de la información histórica y social del hombre, el lenguaje permite la asimilación de toda la información y la experiencia que fueron producidas a lo largo de la práctica histórico-social; esto dio al hombre la capacidad de dominar un conjunto ilimitado de conocimientos, habilidades y formas de comportamiento que no podrían ser desarrolladas por un individuo que se encontrase en un ambiente aislado. El lenguaje representa, en el hombre, un nuevo tipo de desarrollo psíquico, desconocido en los animales, imprescindible para el desarrollo de la conciencia.

El lenguaje trasciende en todos los ámbitos de la actividad consciente del hombre, proporcionando una renovación superior a los procesos psíquicos, reorganizando de manera sólida los procesos de percepción del mundo exterior y dando lugar a la creación de otras leyes a partir de esa percepción. El lenguaje, por tanto, interfiere y transforma en el individuo sus procesos de atención, proporcionándole condiciones de regirla según su voluntad. El lenguaje también modifica los procesos de la memoria humana cuando, apoyado en los procesos del discurso, se convierte en actividad mnemónica consciente, en referencia a la acción de recordar y de organizar lo que debe ser recordado, capacitándole a ampliar su volumen de información en la memoria y la posibilidad de colocarse de manera arbitraria con respecto al pasado, con el fin de seleccionar en éste, a través del proceso de memorización, aquello que le es relevante en una circunstancia dada.

A través del lenguaje, el sujeto se aparta de la experiencia inmediata y da lugar a la imaginación, un hecho que no existe en los animales. A través del lenguaje son constituidas las formas más complejas del pensamiento abstracto y generalizado, las que representan adquisiciones importantísimas en la historia humana, garantía de la transición de la actividad sensorial hacia la racional. Del mismo modo, el lenguaje contribuye para las transformaciones en la reorganización de la experiencia emocional, lo que crea un nuevo nivel de los procesos psíquicos. Citando a Lúria (1979: 84):

Solamente de instruir al hombre con el fin de levantar el brazo o girar la llave en respuesta a una luz roja y no reaccionar con la azul, y surge de inmediato la consolidación de esta nueva relación. La aparición de cualquier acción ejecutable, basada en una instrucción discursiva, dispensa de cualquier refuerzo incondicional (o biológico). 
La actividad consciente del hombre lo diferencia radicalmente de la conducta animal; ésta nos permite percibir su ilimitada plasticidad y el carácter maleable de sus procesos. Lúria, igualmente preocupado por las cuestiones que conducían a la psicología para la comprensión del ser humano como un ser histórico, social y cultural, no desconsideró los procesos fisiológicos y biológicos. Por el contrario, colocaba que la comprensión de los fenómenos de la conciencia del hombre como ser social también estaba relacionada a motivaciones biológicas, ya que el comportamiento no se comprende exclusivamente como un resultado de las condiciones sociales de vida, mas es la suma de esto a las otras posibilidades de comportamiento que se consolidaron a través de la hereditariedad genótipa y, por supuesto, las condiciones orgánicas reales que experimenta el propio individuo. Según Vigotsky:

Como el desarrollo orgánico tiene lugar en un medio cultural, se convierte en un proceso biológico condicionado históricamente. Por otro lado, el desarrollo cultural adquiere un carácter particular e incomparable, ya que se realiza simultánea y fusionadamente con la maduración orgánica, por tanto, su portador resulta ser el organismo del niño que madura, que cambia, crece (1987: 40).

Con tal que el pensamiento y el lenguaje, en principio, no sean iguales,

El hecho principal que encontramos en el análisis genético del pensamiento y el lenguaje es que la relación entre estos procesos no se da en una magnitud constante, e invariable durante todo el desarrollo, mas por una magnitud variable. La relación entre el pensamiento y el lenguaje se modifica en el proceso de desarrollo tanto en lo cuantitativo como en lo cualitativo. En otras palabras, el desarrollo del lenguaje y del pensamiento se realiza de manera desigual y no paralelamente. Las curvas durante ese desarrollo constantemente convergen y divergen, se cruzan o se nivelan e incluso en ciertos momentos siguen en paralelo, llegando a converger en algunas de sus partes para después volver a bifurcarse (Vigotsky, 2000: 111).

Conforme el autor, en una perspectiva dialéctica del desarrollo, la cognición, así como todas las funciones psicológicas superiores, durante el proceso de desarrollo se encuentran inter-relacionadas, por ejemplo, a partir de una revolución, el lenguaje se convierte en lenguaje pensado y el pensamiento pasa a ser verbal, con el fin de operar en la organización del pensamiento, mas también en función de él.

En la esencia de la teoría socio-histórica, en específico en relación al funcionamiento psicológico del ser humano, éste se constituye por la interrelación de diferentes funciones y en todo este proceso el lenguaje desempeña un papel fundamental. Durante sus estudios, Vigotsky, se concentró en el carácter histórico y social de la mente del hombre y en los mecanismos que lo convierten en un ser humano, buscando formar categorías y conceptos para la construcción de una teoría psicológica que respondiese a las preguntas sobre la psique humana desde una perspectiva dialéctica. Es en esta búsqueda que surge el concepto de la mediación.

La acción del hombre tiene impacto de desarrollo por encontrarse bajo la dependencia de la creación de condiciones técnicas y semióticas. Es por medio de la actividad humana que el hombre produce, modifica la naturaleza y la establece en objeto de su conocimiento, y al mismo tiempo se convierte a sí mismo en sujeto de ese conocimiento. La relación del sujeto es dialéctica y está mediada por la semiótica, siendo que los medios técnicos y semióticos son derivados del ámbito social y cultural. 
En ese proceso, los objetos son comprendidos por representaciones e imágenes sensoriales que son inherentes al propio objeto. Para la transposición entre lo que es propio del objeto, su generalización y abstracción, la imagen debe ser simbolizada por un signo repleto de significados de origen cultural y social. Siendo así, un niño puede percibir una comprensión del objeto semiótico porque la imagen está relacionada a un significado, a partir del uso de la palabra. Por lo tanto, la representación simbólica tiene tanto la función de mostrar algo que no se encuentra presente, como la de representar al objeto real en su significado. La palabra es el signo que tiene la función de señalar el objeto y de dar significado al concepto de ese objeto, caracterizándose esta como un instrumento del pensamiento. Tal como afirma Vigotsky (2000:398), "encontramos en el significado de la palabra, a la unidad que refleja de la forma más simple, la unidad del lenguaje y del pensamiento."

\section{EL PENSAMIENTO Y EL LENGUAJE SEGÚN VIGOTSKY}

De esta forma, podemos observar la existencia de la relación entre la actividad productiva del ser humano y su actividad cognitiva, ya que la actividad productiva implica la transmutación del conocimiento histórico producido por la humanidad en conocimiento personal o individual, proporcionando, a través de la actividad cognitiva, la apropiación de los conocimientos producidos por la historia, y por las formas de conocimiento y pensamiento adquiridas por el propio individuo. Es en este proceso de apropiación cultural que se encuentra el lenguaje como mediador y formador de la conciencia.

En la relación entre el pensamiento y el lenguaje, Vigotsky (1987:108-109) destaca la relación que existe entre el pensamiento y la palabra:

La relación entre el pensamiento y la palabra no es una cosa sino un proceso, un movimiento
continuo de ida y vuelta entre el pensamiento y la palabra y viceversa. Durante este proceso,
la relación entre el pensamiento y la palabra pasa a través de cambios que, en sí mismos,
pueden ser considerados un movimiento en sentido funcional. El pensamiento no es simple-
mente expresado por palabras, mas es a través de ellas que pasa a existir [...] El pensamiento
pasa por varias transformaciones hasta convertirse en habla. No es sólo su expresión lo que
encuentra en el discurso, mas encuentra su realidad y su forma.

Para Vigotsky el pensamiento es el reflejo generalizado de la realidad que se concretiza por medio del lenguaje, que está siempre asociado al pensamiento en general, diferenciándose de la palabra que trae consigo un rasgo de abstracción. Por ejemplo, la expresión de un pensamiento se da por medio de diferentes palabras, sin embargo, el pensamiento engloba de una sola vez la acción pensada, su contexto, color, ubicación, etc. De esa manera, tornando existentes los objetos y acontecimientos de la realidad a través del uso de la palabra, el ser humano va más allá de la percepción y de las sensaciones inmediatas, pasando inclusive a concebir que todo lo que piensa está más allá de lo que realmente puede percibir. O sea, la acción de la formación de ideas mediante la palabra da la posibilidad de alcanzar lo que suele ser incomprensible para la capacidad de percepción y para la representación. 
Una palabra no se refiere a un solo objeto, sino a un grupo o a una clase de objetos, por lo que cada palabra ya es una generalización. La generalización es un acto verbal del pensamiento y refleja la realidad de una manera bien diferente a la sensación y la percepción. Esta diferencia está implícita en la idea de que hay un salto dialéctico, no sólo entre la ausencia total de conciencia (en la materia inanimada) y la sensación, sino también entre la sensación y el pensamiento (Vigotsky, 1987: 4).

Por lo tanto, es a través de la generalización que los seres humanos pueden sacar conclusiones acerca de aquello que no perciben de inmediato, yendo más allá de la percepción real, para un analice de mayor rigor y profundidad, a partir de la generalización que se encuentra en los objetos y en los fenómenos que son el resultado de las asociaciones reales y fundamentales entre sí. Es del pensamiento que emanan los conocimientos adquiridos por los seres humanos en su relación con el mundo natural y cultural, estando unido de forma dialéctica al conocimiento sensorial.

Para Vigotsky, el proceso de adquisición de la lengua en el habla infantil tiene sus raíces en el proceso social, a partir del ambiente externo, para convertirse poco a poco en un sistema de signos. Cuando esto sucede, el habla del niño se distingue en dos sistemas de pensamiento que se encuentran extremos, mas, al mismo tiempo, se complementan. Mientras uno de esos sistemas busca adaptarse al mundo exterior, surgiendo como discurso social de adultos, el otro comienza su internalización, convirtiéndose progresivamente en un lenguaje personal. En ese proceso de discurso interior, el sentido de la palabra supera al significado de la propia palabra. Es importante destacar que, según Vigotsky, existe una diferencia entre el significado y el sentido. Citando a Pino (2000: 39):

Para él, el sentido es la suma de los eventos psicológicos que la palabra evoca en la conciencia. Es un todo fluido y dinámico, con zonas variables de estabilidad, una de estas zonas, la más estable y precisa, es el significado. Ésta es una construcción social de origen convencional (o socio-histórica) y su naturaleza es relativamente estable. Los cambios del sentido no afectan la estabilidad del significado. Según Vygotsky, las palabras adquieren su sentido en el contexto del discurso. El cambio de contexto implica, por lo tanto, en cambio de sentido.

El lenguaje es, por tanto, un instrumento de la conciencia con el atributo de estructurar, controlar y planear el pensamiento en una función de intercambio social. Los significados de las palabras constituyen la conciencia del individuo, al mismo tiempo que se constituyen en el contexto interindividual. Así, percibimos la existencia de las relaciones de interdependencia entre el pensamiento y el habla, entre el diálogo interior y el exterior, entre el sentido y el significado, entre el hombre y el mundo que le rodea.

Para Vigotsky, la formación de la conciencia y el desarrollo cognitivo se producen de fuera hacia dentro, siguiendo un proceso de internalización no mecánico, determinado por la actitud del sujeto. Ese proceso de transformación posibilita la construcción del conocimiento y de la cultura y se relaciona a una actividad mental que representa el dominio de los instrumentos de mediación del hombre con el mundo. Por lo tanto, "el pensamiento y el lenguaje son la llave para la construcción de la naturaleza de la conciencia humana” (Vigotsky, 2000: 485).

Los medios de comunicación culturales -en este caso específico, el habla- no son externos a nuestra mente, todo lo contrario, ellos se desarrollan en ella. Así, un niño que llegó a dominar el lenguaje como una herramienta cultural, nunca más será el mismo. Por lo tanto, la cultura y la mediación semiótica constituyen el eje de la teoría de Vigotsky sobre el funcionamiento mental del hombre y esta se relaciona con la existencia concreta 
del sujeto en su proceso social, al mismo tiempo en que es producto de su vida y de sus actividades sociales.

La mediación semiótica, por su vez, en lo que concierne a su teoría, permite la explicación de los procesos de objetivación y de internalización, las relaciones entre el pensamiento y el lenguaje y la interacción entre el sujeto y el objeto de conocimiento. Es por medio de la actividad mediada que el sujeto se encuentra activo y construyendo su propio conocimiento.

En el abordaje histórico-cultural, la mediación semiótica es esencial para la interiorización de signos; la palabra, por su vez, es el elemento principal y más común entre el locutor y el interlocutor, y siempre se encuentra llena de contenidos o sentidos. Según Vigotsky (1987: 104):

El significado de una palabra representa un amalgama tan estrecha del pensamiento y el lenguaje, que es difícil decir si se trata de un fenómeno del habla o un fenómeno del pensamiento. Una palabra sin significado es un sonido vacío, el significado, por lo tanto, es un criterio de la "palabra", su componente indispensable.

La palabra está presente en todas las acciones de comprensión y de interpretación, de modo que todos los signos, inclusive los no-verbales y que no son transmitidos mediante la oratoria, también se encuentran apoyados en las palabras y se unen a ellas. La palabra no es algo poseído por el individuo, más bien es la mediación de circunstancias interactivas diversas.

La mediación semiótica, que es fundamentalmente humana, hace imprescindible el diálogo. Este no debe entenderse sólo como voces que se suceden, sino como un encuentro discursivo en un espacio y tiempo donde lo social y lo histórico están presentes. En la concepción vigotskiana, son las relaciones sociales y el lenguaje, los constituyentes de la actividad mental. Según Pino:

El concepto de mediación semiótica en la perspectiva y las dimensiones aquí propuestas es un buen instrumento conceptual para pensar acerca de la psique humana como un proceso permanente de producción que involucra el individuo y su entorno socio-cultural en una constante interacción. Este proceso nos muestra no sólo el origen social de las funciones psíquicas como también la naturaleza semiótica de la actividad psíquica (2000: 42).

La teoría histórico-cultural apunta también a la mediación semiótica a través del signo y la interacción con el otro como elementos constitutivos del sujeto. La palabra es la que va constituyendo las relaciones entre las personas, estableciendo normas, conceptuando lo que es cierto y lo errado, definiendo las verdades y mentiras, delimitando los discursos, distinguiendo lo normal de lo anormal, lo que es entendido como deficiente y lo que no es. Enfoca que el hombre participa en una realidad social sólo a través de la interacción entre los individuos, y que esta realidad es mediada por los signos. Apunta horizontes de naturaleza práctica, orientando argumentaciones tanto para el niño que es considerado "normal" como para el "anormal".

En el abordaje histórico-cultural, Vigotsky (1998:73) enfoca la función de los instrumentos y los signos que actúan como mediadores de la acción del hombre en el mundo, siendo el instrumento utilizado en la transformación de la naturaleza y el signo como regulador del comportamiento individual. "El control de la naturaleza y el control del comportamiento están estrechamente vinculados, así como la alteración provocada por el hombre sobre la naturaleza altera la propia naturaleza del hombre." 


\subsection{EL PAPEL DE LOS SIGNOS}

Vigotsky contribuye para destacar la importancia de la creación y el uso de los signos en la constitución de la especie humana, destacando al lenguaje como la pieza principal, inclusive para la expresión del pensamiento: "la transmisión racional e intencional de la experiencia y del pensamiento para el otro, requieren de un sistema de mediación, cuyo prototipo es la oratoria humana, oriunda de la necesidad de intercambio de informaciones durante el proceso de trabajo". (Vigotsky, 1987: 5) Esta implica factores que determinan el desarrollo de la conciencia que, a su vez, permiten la discriminación y la conservación de un objeto en la memoria a través de un determinado nombre, realizando la transición de lo sensorial para lo racional en la representación del mundo. Como complemento, Kassar comenta que:

Es en un mundo determinado (en contacto con el otro) que el sujeto nace, crece, se desarrolla y se constituye. Es en ese mundo que será, para él, interiorizado el proceso de su constitución social. (...) En la ontogénesis, cada nuevo ser humano pasa por procesos de apropiación de signos y, por tanto la utilización de esos signos externos, a través de la apropiación individual, se van convirtiendo en los procesos internos de mediación. Los procesos de mediación se van constituyendo a lo largo del desarrollo; éstos no están presentes en los niños al nacer, y sufren, por lo tanto, transformaciones a lo largo de la vida de cada persona. Este proceso continuo se conoce como internalización. El contacto con el otro - con el mundo humano permite el desarrollo de la cultura. (...) De esta forma, los sistemas simbólicos se desarrollan y organizan los signos en estructuras complejas y articuladas. El proceso de significación marca toda la actividad humana (1999: 69-70).

Podemos notar que las señales que evidencian la sociedad son utilizadas como constitutivas del sujeto, haciendo de los signos parte de los principios básicos de mediación que alteran y transforman el desarrollo humano, de la misma manera que los instrumentos creados por el hombre. El lenguaje, por lo tanto, proporciona la constitución de la actividad psicológica, la transformación y el desarrollo del pensamiento, siendo constitutiva para el hombre y permitiéndole su interacción social, la internalización y la generalización de los significados.

Para tales procesos contribuye el aprendizaje, que ocurre a través de la transformación constructiva de pensamientos, sentimientos y acciones, envolviendo una interacción entre los conocimientos preliminares y los nuevos conocimientos que construyen otros significados psicológicos, que darán lugar a otras acciones, pensamiento y lenguaje.

\subsection{EL APRENDIZAJE Y LA FORMACIÓN DE CONCEPTOS}

En cuanto al desarrollo y el aprendizaje, Vigotsky dice que se trata de factos distintos y relacionados, que se hace necesario considerar el nivel de desarrollo ya alcanzado y también el nivel de desarrollo proximal, vinculado a la capacidad de resolución de problemas, a partir de la ayuda de otras personas que se encuentren mejor posibilitadas, indicando que el niño podrá ser autónomo en el futuro, cuando su nivel de desarrollo lo permitir.

El aspecto más esencial de nuestra hipótesis es la noción de que los procesos de desarrollo no coinciden con los procesos de aprendizaje. O más bien, el proceso de desarrollo avanza más lentamente y detrás del proceso de aprendizaje, de esta secuencia, es que resultan las zonas del desarrollo proximal [...] Aunque el aprendizaje esté directamente relacionado con el 
curso del desarrollo del niño, los dos no se llevan a cabo en la misma medida o en paralelo. El desarrollo de los niños no acompaña el aprendizaje escolar de la misma manera que la sombra acompaña al objeto que la proyecta. De hecho, existen relaciones dinámicas muy complejas entre los procesos de desarrollo y de aprendizaje que no pueden ser abarcadas por una formula hipotética inmutable (Vigotsky: 1994: 118-119).

Vigotsky, estudiando la cuestión del aprendizaje y su influencia en el proceso de desarrollo mental, elaboró el concepto de la Zona de Desarrollo Proximal (ZDP), la cual explica como: "La distancia entre el nivel de desarrollo real, que es determinado a través de la resolución independiente de problemas, y el nivel de desarrollo potencial, determinado a través de la resolución de problemas bajo la orientación de un adulto o con la colaboración de compañeros más capacitados" (Vigotsky, 1984: 97).

En relación a las actividades escolares, la acción mediada en la Zona de Desarrollo Proximal despierta diversos procesos internos y ejecuta diversas funciones y procesos que hasta entonces no estaban maduros en el alumno, auxiliando al maestro, agente de la mediación, como una herramienta importante en su trabajo que lleva en cuenta las mediaciones histórico-culturales presentes en los contextos y las situaciones escolares.

El maestro, en su relación con el estudiante, lo lleva a la aprensión de significados adoptados y conceptos elaborados, además de valerse de instrumentos y del propio lenguaje en el proceso de enseñanza y de aprendizaje, haciendo que el conocimiento sea más accesible. Él actúa como un agente de la mediación entre el contacto de su alumno y la cultura que es desarrollada en la relación con los otros, proporcionando la adquisición del conocimiento a partir de diversas circunstancias que generan una comprensión significativa.

Para él, el niño está expuesto a innúmeros signos -que necesitan ser interpretados- y a los significados dentro de un contexto organizado por creaciones simbólicas inherentes a una cultura determinada. Pues, además de las condiciones genéticas o neurológicas que son indispensables en la transformación del individuo, existe también una solicitud del medio cultural, que se espera ofrezca al niño el desarrollo de perímetros complejos y alternativos de comunicación para la incorporación de su contenido, la cultura, las habilidades y los conocimientos construidos por el hombre en la sociedad. Esos perímetros de comunicación progresan en la misma proporción que los perímetros neurológicos, y permiten el desarrollo gradual del pensamiento y del lenguaje como condiciones básicas para el individuo adquirir el significado del mundo que le rodea.

A partir de la Zona de Desarrollo Proximal, las actividades escolares pasan a tener una estrecha relación con la comprensión cualitativa del desarrollo social humano y de los propios contextos escolares a través del desarrollo de las habilidades y capacidades que se construyen en el estudiante con la ayuda de otras personas con más experiencia, en este caso, los profesores. Después de ser internalizadas, tales habilidades y capacidades se convierten en conquistas independientes del niño. También es importante destacar que las interacciones establecidas entre el niño y el profesor no se caracterizan por una mediación estricta y armoniosa, por el contrario, pueden tener lugar en medio de tensiones y conflictos, por lo que la mediación debe ser entendida como un proceso complejo. Sobre este respecto, Vigotsky apunta que:

La formación de conceptos es el resultado de una actividad compleja en la que todas las funciones intelectuales básicas participan. Sin embargo, el proceso no puede ser reducido a la asociación, a la atención, a la proyección de imagen, a la inferencia o a tendencias determinantes. Todas son indispensables, más insuficientes sin el uso del signo o de la palabra, pues 
este es el medio de conducción de nuestras operaciones mentales, y que permite controlarlas y canalizarlas hacia la solución de problemas (1987: 50).

El proceso de la formación de conceptos por parte del sujeto es crucial para su conocimiento del mundo, la interpretación de la realidad y sus significados. En este sentido, Vigotsky trae importantes contribuciones para la enseñanza y las actividades escolares en lo que se refiere al desarrollo de la conciencia reflexiva del alumno, ya que estos procesos tuvieron inicio en conceptos provenientes de la infancia, y que las funciones intelectuales básicas se desenvuelven en la pubertad, momento en que lo abstracto trasciende a los significados resultantes de experiencias inmediatas. Sin embargo, cabe destacar que la relación entre el sujeto y el contexto se lleva a cabo de forma interdependiente, dialéctica y contradictoria, estando la apropiación de los significados sujeta a contextos específicos, a las actividades desarrolladas y a la participación de los sujetos.

Vigotsky (1987), en su investigación, divide el proceso de formación de conceptos en tres etapas:

- La primera denominada de "conglomerado vago y sincrético de objetos separados". En esta, el sujeto establece vínculos subjetivos con vínculos reales entre los objetos. Estos objetos se agrupan a partir del significado de una palabra que, mismo que refleje un vínculo objetivo con la cosa nombrada, también refleja un vínculo casual en referencia a las impresiones subjetivas y las percepciones propias del sujeto.

- La segunda etapa, "pensamiento por complejos", tiene lugar cuando los objetos separados se asocian en la mente del individuo, en función de las impresiones subjetivas ocurridas casualmente y por las relaciones reales concretas que existen entre ellos, basadas en los hechos.

- La tercera etapa, "pensamiento por conceptos", ocurre cuando el individuo tiene la capacidad de abstraer y de escoger separadamente los elementos que forman parte de su experiencia, y de resumirlos de manera abstracta para su utilización instrumental en otros contextos concretos. Aquí el contenido de la experiencia puede comenzar a organizarse de forma abstracta, sin necesidad de una relación directa con las impresiones o las circunstancias concretas.

Por lo tanto, de acuerdo con Vigotsky (1987: 66), para la formación de conceptos es necesario observar que:

La función principal de los complejos es la de establecer vínculos y relaciones. El pensamiento por complejos da inicio a la unificación de las impresiones desordenadas: Al organizar elementos discretos de la experiencia en grupos, crea una base para generalizaciones posteriores. Más el concepto desarrollado presupone algo más allá de la unificación. Para formar ese concepto también es necesario poder identificar los elementos y de examinarlos de manera abstracta e independiente de la experiencia concreta de la cual hacen parte. En la formación de conceptos es igualmente importante el poder unir como el separar: La síntesis debe combinarse con el análisis. Operaciones que, en el pensamiento, dada su complejidad no se pueden realizar.

En el proceso de formación de conceptos es necesario tener en cuenta las especificidades y las relaciones que se dan entre los conceptos cotidianos y los conceptos científicos, ya que ambos conceptos interactúan y se influencian uno al otro como partes de un mismo proceso, el desarrollo de la formación de conceptos. En este proceso único, que es bombardeado por distintas situaciones externas e internas, es que entra el aprendizaje como principio para la formación de conceptos en el niño de edad escolar. 
Desde la perspectiva del abordaje histórico-cultural, el estudiante es un sujeto activo en su proceso de formación y desarrollo intelectual, social y afectivo. El profesor desempeña un papel de agente de mediación de este proceso, proporcionando y favoreciendo la interrelación (encuentro /confronto) entre el sujeto (el estudiante) y el objeto de su conocimiento, que es el contenido escolar. Durante ese proceso de mediación, el saber del estudiante, en cuanto sujeto activo, es muy importante para la formación de su conocimiento. La enseñanza es entendida como una intervención intencional, una inferencia en los procesos intelectuales, sociales y afectivos del alumno, y que visa la construcción del conocimiento del mismo como centro de la enseñanza y sujeto de este proceso.

En palabras de Vigotsky:

La palabra nunca se refiere a un único objeto, sino a todo un grupo o clase de objetos. Por lo tanto, cada palabra es una generalización latente, toda palabra generaliza y, en términos psicológicos, es antes de todo una generalización. Más la generalización, como es fácil de percibir, es un acto verbal excepcional del pensamiento, un acto que refleja la realidad de forma completamente diferente de aquella que es reflejada por las sensaciones y las percepciones inmediatas (2000: 09).

El lenguaje, como instrumento del pensamiento, encuentra su unidad con el propio pensamiento en el significado de las palabras. Así, el trabajo con significado da sentido a la realización del proceso de generalización durante la búsqueda de la apropiación del conocimiento por parte del alumno. La formación de conceptos proviene de la generalización en los diferentes niveles conceptuales, o sea, consiste en la organización de un sistema que sigue como criterio el nivel de generalización.

\subsection{LAS CONTRIBUCIONES DE VIGOTSKY PARA LA EDUCACIÓN DE ALUMNOS CON NECESIDADES ESPECIALES EN LA PERSPECTIVA INCLUSIVA}

Con base en los presupuestos del abordaje histórico-cultural aportados por Vigotsky, tenemos una percepción de la realidad educativa que vivimos, la que, a menudo, impide a una persona con necesidades especiales desarrollarse plenamente, sobre todo debido a las conclusiones prejuiciosos que existen sobre su aprendizaje. Sin embargo, si esta persona tuviese acceso al contacto con el otro, y a una orientación pedagógica adecuada y organizada, su desarrollo podría realizarse por el acceso a la cultura que es producida históricamente.

En los presupuestos de Vigotsky, resulta claro que la apropiación del conocimiento es construido de forma histórica y mediada en su relación con el profesor a través del lenguaje que es el cierne de todo lo que es social, que interactúa, que dialoga, que ejerce ciudadanía. Igualmente, éstos rechazan las posiciones dicotómicas de la época relativas a la psicología del lenguaje, que se caracterizaba por el racionalismo y el empirismo. En búsqueda de una concepción de psicología que comprendiese al ser humano como un todo, y una educación que resguardase el espacio individual del sujeto, enfatiza que el sentido para el sujeto se construye en el lenguaje y que en éste se encuentran incluidos, en todo momento, el diálogo y la interacción social con el otro.

De esta forma, comprendemos la necesidad de reflexionar como personas y profesionales sobre nuestra propia constitución en cuanto a sujetos para, finalmente, elaborar y trabajar dentro de un abordaje que releve la historia, la cultura y lo social como aspectos esenciales en la constitución de la persona, ya sea que se trate de un niño con 
autismo,por ejemplo, lo que nos lleva a concluir que el factor biológico, centrado en las ciencias naturales, no puede ser el factor determinante para su desarrollo. Por cuanto, la organización y la construcción de la actividad mental no se encuentran en el interior del sujeto, mas sí en la interacción verbal mediante la cual es generada la expresión que organiza tal actividad de manera relativa a su nivel de ajustamiento social.

En relación a las ideas de Vigotsky, son percibidas las intenciones de una concepción de lenguaje que releve los diversos sentidos que una determinada palabra puede encontrar en la amplitud entre lo verbal y lo no verbal, en que el movimiento de elaboración de la psique humana sea la prioridad delante de los condicionamientos institucionales. Eso propiciaría el desarrollo de una propuesta pedagógica no-reduccionista de la potencialidad del niño con necesidades especiales y que se aparta de los atendimientos de base behaviorista y de la acentuación de manierismos producidos por las actuaciones mecanicistas, centradas en el estímulo-respuesta dado por los diversos profesionales que están en contacto con este niño, pues el lenguaje debe ser comprendido como un espacio que propicie la recuperación del individuo, en cuanto sujeto, como ser histórico y social.

Teniendo en vista la importancia del lenguaje, cabe aquí que coloquemos la razón que nos torna en seres sociales. Las características del ser humano se desarrollan en el medio en que se desenvuelve la vida del propio hombre, así ocurrió en los pueblos primitivos y ocurre también hoy, mismo considerando la evolución del propio cerebro, en el propósito de resolver problemas más complejos. Por lo tanto, el lenguaje, una vez más, nos muestra su papel memorable, ya que la comunicación que se da a través de este y de la emoción, constituye un componente esencial para la evolución y la adaptación del hombre.

Así, a pesar de que la deficiencia pueda provocar alteraciones temporales o permanentes y que, en su recorrer puedan surgir incapacidades comprometiendo el desempeño y la actividad funcional de la persona, lo que implica en desventajas para la adaptación y la interacción social, se podrán encontrar posibilidades de compensación para conseguir un desarrollo psicológico más significativo en estos casos. Dicha compensación dependerá de la existencia de relaciones sociales y de las mediaciones semióticas que permitan la superación de los déficits.

La peculiaridad positiva del niño con deficiencias también se origina, en primer lugar, no porque en él desaparecen unas u otras funciones observadas en un niño normal, sino porque esta desaparición de las funciones hacen que surjan nuevas formaciones que representan, en su unidad, una reacción de la personalidad ante la deficiencia, la compensación en el proceso de desarrollo. Si un niño ciego o sordo alcanza en el desarrollo lo mismo que un niño normal, entonces los niños con otras deficiencias lo alcanzan de un modo diferente, por otra vía, con otros medios y para el pedagogo es muy importante conocer la peculiaridad de la vía por la cual él debe conducir al niño. La ley de la transformación del menos de la deficiencia en el más de la compensación proporciona la clave para llegar a esa peculiaridad (Vigotsky, 1989: 7).

Del mismo modo, en relación a la competencia social del individuo -que envuelve la contribución de todo el cerebro- según las investigaciones actuales, el cerebelo que coordina y recibe constantemente informaciones visuales, auditivas y somatosensoriales, también parece coordinar algunos procesos mentales como la cognición y la atención, lo que implica en el funcionamiento y el desarrollo del comportamiento social, e inclusive en la mediación cognitiva. Según Vigotsky (1989: 32-33): 
La educación de los niños con diferentes defectos debe basarse en el hecho de que simultáneamente con el defecto estén dadas también las tendencias psicológicas de una dirección opuesta; estén dadas las posibilidades de compensación para vencer el defecto y de que precisamente esas posibilidades se presentan en primer plano en el desarrollo del niño y deben ser incluidas en el proceso educativo como su fuerza motriz. Estructurar todo el proceso educativo según la línea de las tendencias naturales a la súper compensación, significa no atenuar las dificultades que surgen del defecto, sino tensar todas las fuerzas para su compensación, presentar solo las tareas y en un orden que respondan al carácter gradual del proceso de formación de toda la personalidad bajo un nuevo punto de vista.

En el caso de las personas con necesidades especiales, desde la perspectiva de desarrollo de la educación tradicional, que está centrada en los síntomas o la enfermedad, las condiciones comúnmente encontradas implican en dificultades de aprendizaje, de comunicación y de interacción, lo que genera cierta complejidad en relación con la labor a ser realizada por el educador. Sin embargo, lo mismo en tales circunstancias, en la perspectiva del abordaje histórico-cultural se espera que estos individuos den un salto a partir del contexto de las relaciones personales de las actitudes propicias que facilite el educador y de la acción mediadora de los signos.

El lenguaje, por tanto, para nosotros, en cuanto actividad de la especie humana, puede ser comprendido como cualquier sistema de signos encontrados, en la comunicación social recíproca para dar a entender, conocer y tornar comunes a las representaciones mentales concretas o abstractas, así como a los sentimientos que se pueden entender como conservados en nuestra conciencia, teniendo en cuenta que ésta se construye históricamente en las relaciones sociales del individuo con su entorno cultural. En el abordaje históricocultural encontramos un entendimiento más amplio y una clara expresión acerca de la dialéctica interior y exterior, a partir de la mediación propiciada por la interacción verbal.

\section{CONCLUSIONES}

De acuerdo con la teoría histórico-cultural, la constitución del sujeto no está determinada exclusivamente por factores biológicos. Igualmente, no se puede concebir a una persona con necesidades especiales como la representación de una máquina, ni tan solamente como a un organismo, mas sí como un sujeto social que se construye en las relaciones sociales, culturales e históricas, a través de la mediación de otro sujeto y de los signos existentes en esa mediación.

En esta perspectiva teórica, el lenguaje ejerce un papel y una función excepcional. Se concibe al ser humano como un ser eminentemente simbólico, siendo el lenguaje el responsable del proceso de transformación de las funciones psicológicas interpersonales en intramentales, dando lugar así a la constitución del pensamiento, de la conciencia y de las otras funciones psíquicas superiores, propias de la especie humana. Asimismo, se concluye que el abordaje histórico-cultural de Vigotsky ofrece contribuciones de significativo valor en lo que respecta a la educación de personas con necesidades especiales dentro de una perspectiva inclusiva. 


\section{REFERENCIAS BIBLIOGRÁFICAS}

Kassar, M. C.M. (1999). Deficiência múltipla e educação no Brasil. São Paulo: Autores Associados. Luria, A. R. (1979). Curso de Psicologia Geral: Introdução Evolucionista à Psicologia. Vol. I. Rio de Janeiro: Civilização Brasileira. Médicas.

(1987). Pensamento e linguagem: as últimas conferências de Luria. Porto Alegre: Artes

Pino, A. (2000). O conceito de mediação semiótica em vygotsky e seu papel na explicação do psiquismo humano. Caderno CEDES, n. 24, 32-43.

. (2000). O social e o cultural na obra de Vigotski. Educação \& Sociedade, a. XXI, n. 71, 45-78.

Vigotsky, L.S. (1998). A formação social da mente. São Paulo: Martins Fontes. . (1987). Pensamento e Linguagem. São Paulo: Martins Fontes.

. (1989). Obras Completas - Fundamentos de Defectología. Ciudad de La Habana, Cuba: Editorial Pueblo Y Educación.

. (2000). A construção do pensamento e da linguagem. São Paulo: Martins Fontes.

Vygotsky, L. S., Luria, A .R. y Leontiev, A. N. (1988). Linguagem, Desenvolvimento e Aprendizagem. São Paulo: Ícone.

. (1987). Historia del desarrollo de las funciones psíquicas superiores. Havana, Cuba: Científico-Técnica, 1987. 